\title{
BUSINESS CONTINUITY PLANNING AND LEARNING BASED ON AN EXTENSION TO THE JDL DATA FUSION MODEL
}

\author{
Sami Mohammed ${ }^{1}$, Sabah Mohammed ${ }^{2}$ and Jinan Fiaidhi ${ }^{2}$ \\ ${ }^{1}$ Department of Computer Science, University of Victoria, Canada \\ ${ }^{2}$ Department of Computer Science, Lakehead University, Canada \\ ${ }^{1}$ smohamm@uvic.ca, ${ }^{2}\{$ jfiaidhi,mohammed\}@lakeheadu.ca
}

\begin{abstract}
Small businesses are likely to suffer severely from any disaster. Only half of businesses had any kind of plan in place to recover from such unforeseen events. The pain of disaster will be felt not only by the businesses themselves, but by their customers. Not surprisingly, this lead to a lot of customer loss where they will switch vendors due to "unreliable business systems". Business continuity planning (BCP) is the process of creating systems of prevention and recovery to deal with potential threats to a company. This summary paper presents an extended model of JDL BCP by adding two essential levels including machine learning and workflow integration. The Node-Red workflow integration platform is suggested to be used for BCP prototyping and recovery.
\end{abstract}

Keywords - BCP Modeling, JDL Model, Node-Red, Planning and Recovery

\section{INTRODUCTION}

Business Continuity Planning (BCP) is a proactive planning process that ensures critical services or products are delivered during a disruption. A Business Continuity Plan includes [1]:

$>$ Plans, measures and arrangements to ensure the continuous delivery of critical services and products, which permits the organization to recover its facility, data and assets.

$>$ Identification of necessary resources to support business continuity, including personnel, information, equipment, financial allocations, legal counsel, infrastructure protection and accommodations.

Having a BCP enhances an organization's image with employees, shareholders and customers by demonstrating a proactive attitude. Additional benefits include improvement in overall organizational efficiency and identifying the relationship of assets and human and financial resources to critical services and deliverables. BCP involves defining any and all risks that can affect the company's operations, making it an important part of the organization's risk management strategy. Risks may include natural disastersfire, flood, or weather-related events - and cyber-attacks. Once the risks are identified, the plan should also include [2]:

$>$ Determining how those risks will affect operations

$>$ Implementing safeguards and procedures to mitigate the risks

Received: July 5, 2019

Reviewed: October 15, 2019

Accepted: October 29, 2019

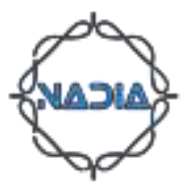


Testing procedures to ensure they work

$>$ Reviewing the process to make sure that it is up to date

BCPs are an important part of any business. Threats and disruptions mean a loss of revenue and higher costs, which leads to a drop in profitability. And businesses can't rely on insurance alone because it doesn't cover all the costs and the customers who move to the competition. There are several legacy techniques where most of the companies follow to develop kind of a BCP. They include:

$>$ Business Impact Analysis: is to identify functions and related resources that are time-sensitive.

$>$ Recovery Investigation: is to identify and implement steps to recover critical business functions.

$>$ BCP Team Creation: A team must be created to devise a plan to manage the disruption.

$>$ Training and Testing: The continuity team must be trained to go over the plan and strategies and test them through series of exercises.

With BCP systems, the underlying principle is the creation and maintenance of a realtime and accurate model of the world. As part of these systems, situational assessment (SA) is an important component as it combines the numerous data sources, interfaces to the user and manages data collection and information extraction. In order to ensure the presentation of a reliable and coherent situational picture to the stakeholders, the $\mathrm{BCP}$ system must not only be able to fuse these data streams in real-time, issuing alerts when anomalous behavior or a disaster is detected, but also mine the raw data for patterns that represent information and storing the set of patterns in the knowledge base being maintained by the system. Fusion is all about delivering management software and solutions for organizations to achieve enterprise resilience aligned to executive's fiscal and fiduciary responsibility, customer commitments, regulatory requirements and stockholders expectations [3]. Using Fusion Technologies and Models enterprises are provided with a greater operational resilience by supporting the full risk and continuity spectrum that today's businesses need. The fusion platforms eliminates the need for separate modules across the many areas of risk and continuity management, making it easy to expand into new areas when ready, operating as a completely integrated program on a common information foundation. However, the use of a representative fusion model is important to accomplish accurate risk assessment. There are four fusion models that have been taken as a lingua franca for data fusion problems including those used for business continuity planning and learning:

- JDL model $[4,5]$

- DDF model [6]

- Omnibus model [7]

- Perceptual Reasoning model [8]

However, none of these models deal with multiple data of different types as well as account for human processing. All these models performs automatic processing of signals from machines and does not account for human interactions. In a typically fusion architecture one need to support the role and interactions of users. In this paper we are proposing an extension to JDL Model where it can support different types of data integration and user interactions as intrinsic part of the fusion system for business continuity 


\section{EXTENDING THE JDL FUSION MODEL FOR BUSINESS CONTINUITY PLANNING}

The Joint Directorate Laboratories (JDL) which was researched in 1993 came up with a multi-level data fusion model, primarily focused on military applications. The idea was that you could look at the state of the world considering different entities as "atomic units" of your "World View". Level 1 Fusion, for example, was called "Object Refinement" and was focused on fusing multiple heterogeneous data sources to obtain information about individual objects (e.g., people, vehicles, buildings, etc.). So-called Higher Level Fusion dealt with Levels 2-4, which were named "Situation Refinement", "Threat Refinement", and "Process Refinement". Note, there is a big conceptual jump from Level 1 to Level 2. Level 1 consists of real, measurable objects that exist in time and space. The other levels are concepts and exist in "linguistic space" instead of physical space. In 1998, the JDL model was upgraded to more general, less militaristic language. There was also a "Level 0" added. So the levels now were "Sub-Object Assessment", "Object Assessment", "Situational Assessment", "Impact Assessment", and "Process Refinement". Sub-Object Assessment referred to using data to resolve things smaller than individual objects. So it could be an arm, or it could refer to integrating ("fusing") consecutive reflected pulses from a radar to form a signal [9]. In 2015, a researcher [10], extended the four levels JDL model into five level one where Level 5: "User Refinement" involves humans to be "in the loop" affecting the products of all the lower levels.

However, the increasing amount of information available for business continuity planning motivates the adoption of machine learning methods for addressing specific situation assessment or risk. A special focus need to be placed on prognosis, namely, the capability to estimate and anticipate events of interest regarding assets and production processes. There lies indeed at the core challenge of business continuity planning from a data science perspective: data-driven prognostic approaches aim at predicting when an abnormal behavior is likely to arise within the monitored process, providing further insights such as its severity and impact on the business performance. For this reason it becomes particularly interesting to characterize normality properly towards unveiling degradation patterns or trends. Thus adding a sixth level to JDL to include machine learning is one of the most important direction for business continuity planning and learning. The idea of having the sixth level is to characterize behavioral patterns of interest on the basis of the data monitored from the process or asset under study (training data) by means of machine learning models. This acquired knowledge can be then applied to new unseen data (test data) to tackle a wide variety of planning problems, including prediction, classification and anomaly detection, among others. Furthermore, since data and services are the most important asset that a business owns, and in today's climate of data security and stewardship it's more important than ever to trace how that data was produced and the journey it has undertaken through the workflow of services leading to its present state. What we need in this direction is a strong data and services governance platform. This platform represent another architectural layer (seventh level) to the JDL model where the layout of the BCP system is developed as a suite of small services, each running in its own process and communicating with lightweight mechanisms. The benefits of level 7 are many, ranging from an increase in development productivity, to better business-IT alignment, agility, scalability, and technology flexibility. Figure 1 illustrate a schematic view of our proposed extended JDL model with addition of the two new levels of 6 and 7. 


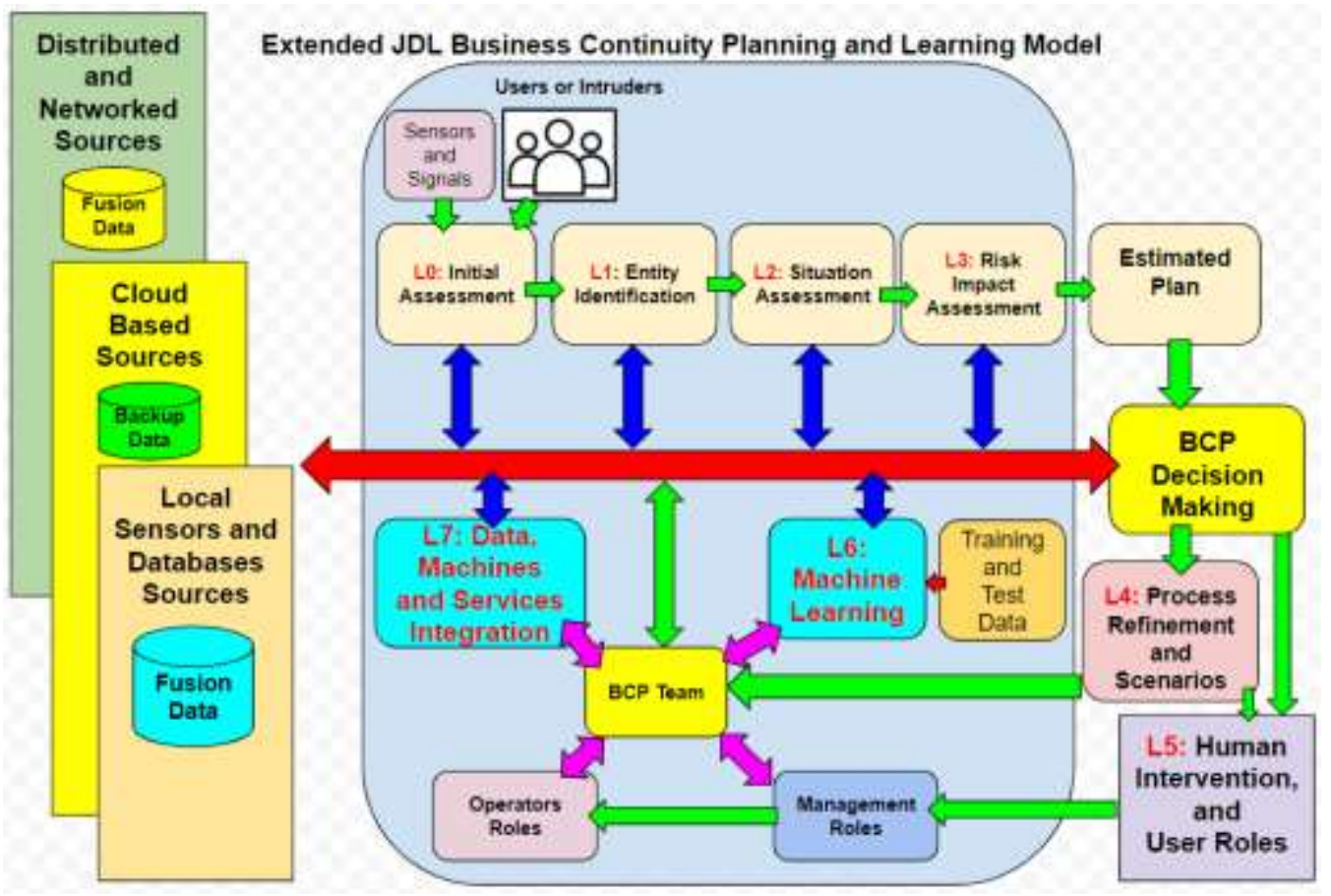

Fig. 1 Extended JDL Model for Business Continuity Planning and Learning.

In order to contain our illustration on the extended JDL model, we focus on level 7 in which a business system is observed for the specification, aggregation, and evaluation of the quality and risk attributes for the services workflows and data integration. Level 7 allows developers to (i) produce architecture models of the system, either manually or automatically via recovering techniques, (ii) contribute to an ecosystem of well-specified services workflows and integration, and (iii) continuously measure and evaluate the $\mathrm{BCP}$ architecture of their systems in the ecosystem.

\section{BCP DATA, MACHINES AND SERVICES INTEGRATION FRAMEWORK}

When it comes to prototyping BCP systems, Node-RED is an obvious choice. This flow-based system originally built by IBM enables you to graphically create a flow which will acquire, process and store your data as well as integrate variety of web services. Node-RED has many third party contributions which make it easy and fast to achieve almost any integration task. Workflows offer developers a simple but flexible programming model at a level of abstraction closer to the domain-specific activities that they seek to perform. However, languages for describing workflows tend to be highly complex, or specialized towards a particular domain, or both. There are a large number of workflow systems designed to work in various scientific domains, including support for the Internet of Things (IoT). Node-RED, which is originally designed to bring workflowbased programming to IoT and extreme automation. However, the majority of workflow systems, and specifically systems like Node-RED, are designed to operate in a fixed networked environment, which rely on a central point of coordination in order to manage the workflow [11]. However, our extended JDL level 7 presents an approach to workflow programming which combines the advantages of a declarative workflow description and web services programming. The workflow execution model is based on a formal model of computation over web services. The execution environment is implemented on the basis of a widely adopted runtime platform node.js. Workflow programming benefits from such an approach in multiple ways, including leveraging a large programming ecosystem 
with many developers, reusable software packages and learning resources; elimination of shim nodes from the workflow graph; and increased reusability of workflow processing components. Figure 2 illustrate the notion of using Node-Red for BCP integration.

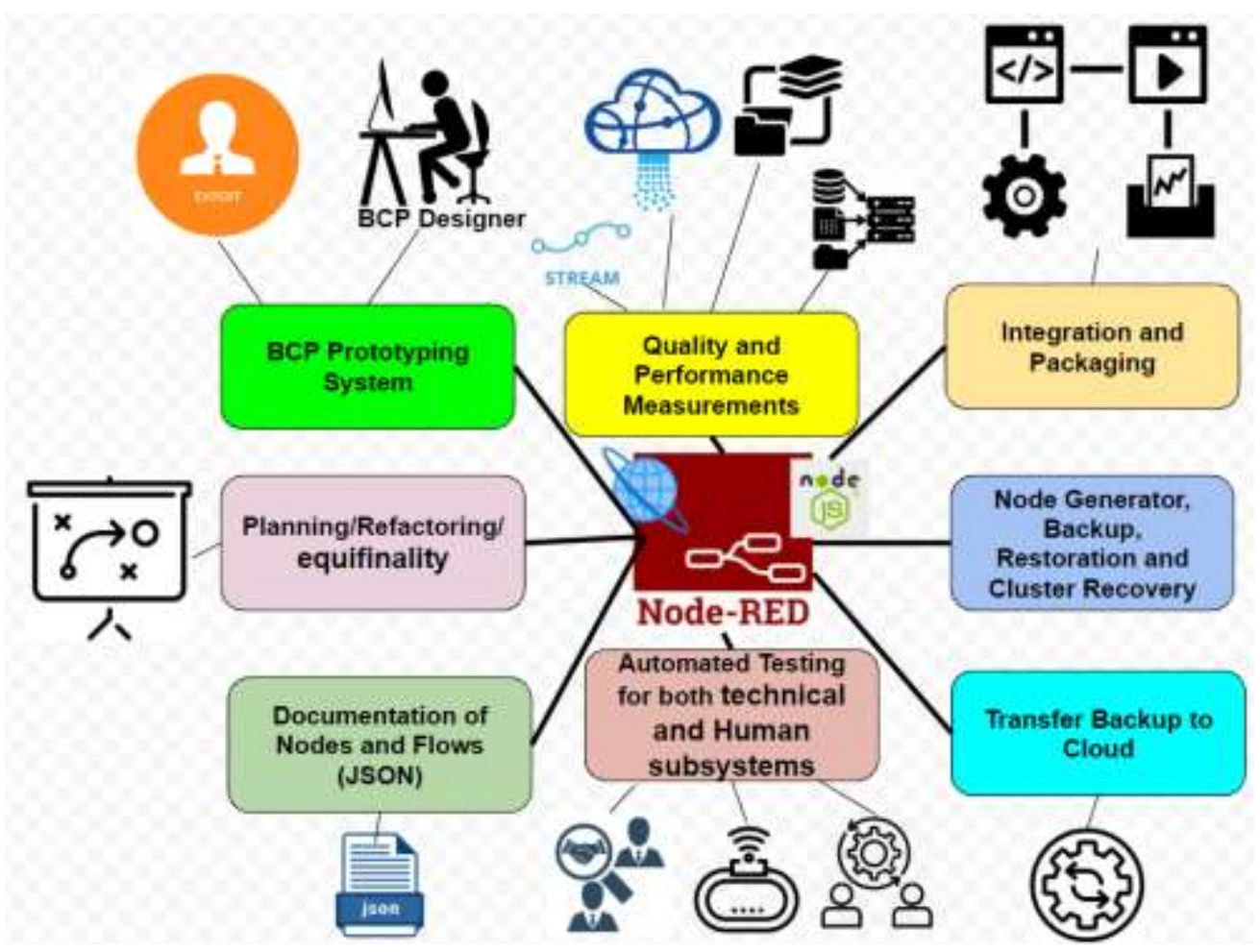

Fig. 2 BCP Integration and Recovery Based on Node-Red Workflow Platform.

At this level the BCP prototyping can be started as well as the restoration and recovery of the system to its previous status. Based on Node-Red flows we can add nodes for mirroring, backup and transfer to the fusion cloud workflows database. The authors have described the details on how to use such integration framework for a typical healthcare fusion ecosystem in their recent paper [12].

\section{CONCLUSIONS}

Many business professionals, experts and organizations have turned to the sociotechnical design concepts, theories, models, tools and methods, to redesign healthcare systems and processes and to improve business safety and continuity. Given the complexity of conducting business in the new technological advances, machine learning and workflow integration are considered to have considerable impact on the business interoperability of the different business workflow services. This article described BCP modelling as an extension to the JDL model by adding two additional levels involving machine learning and workflow integration. The integration level uses the Node-Red workflow composition ecosystem. The authors are working on prototyping the extended JDL system as it applied for healthcare BCP fusion modeling.

\section{ACKNOWLEDGMENTS}

This is a summary paper is an extension from the second and third author NSERC Grants applications renewal. 


\section{REFERENCES}

[1] Public Safety Canada, A Guide to Business Continuity Planning, Minister of Public Works and Government Service Report 2018-01-31, Catalogue No. D82-37/2003E-IN, ISBN 0-662-33765-4, Available Online: https://www.publicsafety.gc.ca/cnt/rsrcs/pblctns/bsnss-cntnt-plnnng/index-en.aspx

[2] WILL KENTON, Business Continuity Planning (BCP), Investopedia report, Jun 1, 2019, Available Online: https://www.investopedia.com/terms/b/business-continuity-planning.asp

[3] Shiv Shanker, Fusion Risk Management: Business Continouity Redefined, March 22, 2019, Available Online: https://business-continuity.cioapplications.com/vendor/fusion-risk-management-businesscontinuity-redefined-cid-2835-mid-165.html

[4] Alan Steinberg, Christopher Bowman and Franklin White, "Revisions to the JDL Data Fusion Model," March 1999. Proc. SPIE Vol. 3719, p. 430-441, Sensor Fusion: Architectures, Algorithms, and Applications III, Belur V. Dasarathy; Ed.

[5] James Llinas, Christopher Bowman, Galina Rogova, Alan Steinberg, Ed Waltz and Franklin White, "Revisiting the JDL Data Fusion Model II," December 2004. Available Online: https://pdfs.semanticscholar.org/b183/008f9d63252bd4cc4438cf0bf744bc8e995d.pdf

[6] B. Dasarathy, "Decision Fusion Strategies in Multisensor Environments ", IEEE Transactions on Systems, Man, and Cybernetics, pp 1140-1154, vol. 21, 1991.

[7] M. Bedworth and J. Obrien, "The Omnibus Model: A New model of data fusion?", AES Magazine, April 2000.Available Online: https://pdfs.semanticscholar.org/7595/1a01b0c603774ed99e57d9b427ce55741020.pdf

[8] Kadar, I, "Perceptual Reasoning in Adaptive Fusion Processing," SPIE, 2002, Available Online:https://www.spiedigitallibrary.org/conference-proceedings-of-spie/4729/0000/Perceptualreasoning-in-adaptive-fusion-processing/10.1117/12.477619.short

[9] Mark Meloon, What is an example of data fusion? How does it help in big data? Is it data analysis technique?, Sep 19, 2014, Available Online: https://www.quora.com/What-is-an-example-of-datafusion-How-does-it-help-in-big-data-Is-it-data-analysis-technique

[10] Erik Blasch, One decade of the Data Fusion Information Group (DFIG) model, Proceedings Volume 9499, Next-Generation Analyst III; 94990L (2015) https://doi.org/10.1117/12.2176934. Event: SPIE Sensing Technology + Applications, 2015, Baltimore, Maryland, United States

[11] Simpkin, Christopher, Ian Taylor, Daniel Harborne, Graham Bent, Alun Preece, and Ragu K. Ganti. "Dynamic Distributed Orchestration of Node-RED IOT Workflows Using a Vec-tor Symbolic Architecture." In 2018 IEEE/ACM Workflows in Support of Large-Scale Sci-ence (WORKS), pp. 5263. IEEE, 2018.

[12] Jinan Fiaidhi, Sabah Mohammed and Li Tengyue, Sociotechnical Workflow Approach for Interoperable Healthcare Systems, Springer Proceeding of the ICFeh 2019, Nov 1-3, 2019 China. 